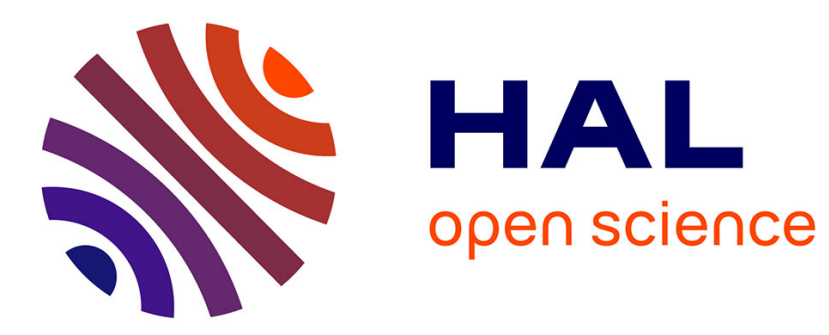

\title{
Capteurs à impulsion pour la caractérisation des transferts thermiques entre une paroi et son environnement microclimatique en régime variable
}

C. Brunjail, F. Fragnaud

\section{- To cite this version:}

C. Brunjail, F. Fragnaud. Capteurs à impulsion pour la caractérisation des transferts thermiques entre une paroi et son environnement microclimatique en régime variable. Revue de Physique Appliquée, 1982, 17 (4), pp.187-199. 10.1051/rphysap:01982001704018700 . jpa-00244988

HAL Id: jpa-00244988

https://hal.science/jpa-00244988

Submitted on 1 Jan 1982

HAL is a multi-disciplinary open access archive for the deposit and dissemination of scientific research documents, whether they are published or not. The documents may come from teaching and research institutions in France or abroad, or from public or private research centers.
L'archive ouverte pluridisciplinaire HAL, est destinée au dépôt et à la diffusion de documents scientifiques de niveau recherche, publiés ou non, émanant des établissements d'enseignement et de recherche français ou étrangers, des laboratoires publics ou privés. 
Classification

Physics Abstracts

$44.25-44.30-86.70 \mathrm{~L}$

\title{
Capteurs à impulsion pour la caractérisation des transferts thermiques entre une paroi et son environnement microclimatique en régime variable $(*)$
}

\author{
C. Brunjail et F. Fragnaud $(*)$
}

I.U.T. de Saint-Nazaire et Laboratoire de Thermocinétique, 44072 Nantes Cedex, France

(*) Laboratoire de Thermodynamique, 44072 Nantes Cedex, France

(Reçu le 26 octobre 1981, révisé le 7 décembre 1981, accepté le 15 décembre 1981)

\begin{abstract}
Résumé. - Dans ce travail, nous avons voulu étudier le principe de capteurs pariétaux permettant de caractériser globalement l'influence des facteurs microclimatiques sur les transferts thermiques superficiels entre une paroi avec revêtement hydrofuge et son environnement en régime variable. Il ressort de cette étude que ces transferts peuvent être caractérisés par la température de paroi $T_{\mathrm{p}}$ et par deux couples de données : soit $h$ et $T_{\mathrm{E}}(h$ coefficient de transfert thermique lié à la paroi, $T_{\mathrm{E}}$ température équivalente) soit deux grandeurs $h_{0}^{\prime}$ et $T_{\mathrm{c} 0}^{\prime}$ directement liés au capteur.

La mesure du couple $h$ et $T_{\mathrm{E}}$ nécessite un capteur avec une régulation délicate; alors que le couple $h_{0}^{\prime}$ et $T_{\mathrm{co}}^{\prime}$ peut être déterminé avec un capteur présentant un simple dispositif de chauffage programmable : $T_{\mathrm{c} 0}^{\prime}$ est obtenu par l'enregistrement de la température d'un capteur non chauffé, et $h_{0}^{\prime}$ par la loi de refroidissement d'un capteur identique au précédent mais chauffé par impulsion. Le coefficient $h_{0}^{\prime}$ lié à la perturbation thermique introduite par le capteur est supérieur au coefficient $h$.

Par des considérations sur la dynamique de l'écoulement, nous retrouvons les corrélations expérimentales entre ces coefficients. L'étude expérimentale des capteurs montre que leur constante de temps est de l'ordre de la minute (elle varie en fonction du coefficient $h_{0}^{\prime}$ de 40 à $80 \mathrm{~s}$ dans le domaine de vitesse d'air exploré). Il est donc possible de suivre des fluctuations microclimatiques de durée égale ou supérieure à $4 \mathrm{~min}$
\end{abstract}

\begin{abstract}
Heat transfers between a wall with waterproof facing and the surrounding atmosphere depends on different microclimatic causes (wind, rain, infra red ray, long wave length, absorbed solar radiation...) which ceaselessly vary in time. Their evolution is difficult to appreciate in a continuous way unless using an expensive experimental arrangement on the very spot. The main aim of the present work is the study and the realisation of parietal heat captors permitting to characterize globaly the evolution of heat transfer under the action of these microclimatic variations.

It comes out of this study that these transfers can be characterized by wall temperature $T_{\mathrm{p}}$ and by two pairs coefficients : as $h$ and $T_{\mathrm{E}}$ as $h_{0}^{\prime}$ and $T_{\mathrm{c} 0}^{\prime}$ ( $h$ coefficient of heat transfert referred to the wall; $T_{\mathrm{E}}$ equivalent temperature; $h_{0}^{\prime}$ coefficient of heat transfert referred to the captor; $T_{\mathrm{co}}^{\prime}$ temperature of the captor).

The measurement of pair $h$ and $T_{\mathrm{E}}$ needs a delicate regulation, where as pair $h_{0}^{\prime}$ and $T_{\mathrm{c} 0}^{\prime}$ can be definied by a captor with a simple programmable heat device : $T_{\mathrm{c} 0}^{\prime}$ is obtained by the temperature acquisition of a captor unheated and $h_{0}^{\prime}$ is obtained by the cooling law of the same captor as the previous one, but heated by impulse. Coefficient $h$ et $h_{0}^{\prime}$ are not equal $\left(h_{0}^{\prime}\right.$ is higher than $\left.h\right)$; they are different in the values of the convection coefficient for the established flow $\left(h_{\mathrm{c}}\right)$ and for the thermal disturbance caused by the captor $\left(h_{\mathrm{c} 0}\right)$. With considerations about flow dynamics we show that $h_{0 \mathrm{c}}$ is directly bound to the coefficient of local friction, hence to speed $u_{\infty}$, of which coefficient $h_{\mathrm{c}}$ is dependent too. The experimental study of these captors shows they are rather sensitive to microclimatic variations and their time-constant is roughly equal to one minute (it varies from $40 \mathrm{~s}$ to $80 \mathrm{~s}$, according to coefficient $h_{0}^{\prime}$ ). Then we can follow with a negligeable delay all the microclimatic variation superior or equal to 4 min., which is very satisfactory. Shorter variations are very poorly energetic and cannot meaningly influence heat transfers.
\end{abstract}

(*) Ce travail a été effectué dans le laboratoire de Thermocinétique de l'U.E.R. de physique de Nantes sous la direction de M. le Professeur Bardon. 
1. Introduction. - Les transferts thermiques entre une paroi et l'ambiance environnante dépendent de divers facteurs microclimatiques (vent, pluie, rayonnement infrarouge, grande longueur d'onde, rayonnement solaire absorbé...) qui fluctuent sans cesse au cours du temps. Leur évolution est difficile à connaître de manière continue à moins de disposer d'un appareillage coûteux sur le site même. Le travail présenté ici, à pour objectif l'étude théorique et expérimentale de capteurs thermiques pariétaux permettant de caractériser globalement l'évolution des transferts thermiques sous l'action de ces fluctuations microclimatiques [1] dans le cas d'une paroi présentant un revêtement hydrofuge.

2. Stylisation des transferts superficiels. - Les transferts de chaleur superficiels résultent :

- de l'absorption de rayonnement solaire $\alpha_{\mathrm{s}} \varphi_{\mathrm{s}}$ $\left(\varphi_{\mathrm{s}}\right.$ : rayonnement solaire incident $; \alpha_{\mathrm{s}}$ absorptivité à ce rayonnement);

- des échanges par rayonnement avec les surfaces environnantes à température radiative $T_{\mathrm{r}}$ Ceuxci, vu les faibles écarts de températures, peuvent se mettre sous la forme :

$$
h_{\mathrm{r}}\left(T_{\mathrm{p}}-T_{\mathrm{r}}\right)
$$

$h_{\mathrm{r}}$ est le facteur de rayonnement, il dépend de l'émissivité des parois, de leurs facteurs de formes et de $T_{\mathrm{m}}^{3}, T_{\mathrm{m}}$ est une température moyenne

$$
T_{\mathrm{m}}=\frac{T_{\mathrm{p}}+T_{\mathrm{r}}}{2}
$$

- des échanges par convection avec l'air ambiant à $T_{\mathrm{a}}$ qui peuvent être stylisés par la relation :

$$
h_{\mathrm{c}}\left(T_{\mathrm{p}}-T_{\mathrm{a}}\right)
$$

$h_{\mathrm{c}}$ est le coefficient de convection;

- dans le cas d'une paroi mouillée, des échanges par évaporation avec l'air non saturé (pression partielle de vapeur $P_{\mathrm{v}}$ ). Ces derniers peuvent, là encore s'expliciter vu le faible écart $T_{\mathrm{p}}-T_{\mathrm{a}}$, par une relation de la forme $: h_{\mathrm{v}}\left(T_{\mathrm{p}}-T_{\mathrm{a}}\right)+\varphi_{\mathrm{v}}, h_{\mathrm{v}}$ et $\varphi_{\mathrm{v}}$ étant proportionnels à la chaleur latente de vaporisation de l'eau, et dépendant de la température de l'air; $\varphi_{\mathrm{v}}$ dépend en plus du degré hygrométrique de l'air. On peut noter qu'il y a analogie entre les transferts de chaleur par convection dans l'air sec et les transferts massiques de vapeur d'eau sous l'action du gradient de concentration; la notion de couche limite s'applique aux deux transferts : transferts thermiques dans l'air, diffusion de la vapeur d'eau dans l'air, et comme le nombre de Schmidt pour ce transfert massique est très voisin du nombre de Prandtl, les épaisseurs des couches limites thermiques et massiques coïncident pratiquement [2].

Finalement, la densité de flux $\varphi_{\mathrm{p}}$ perdue par la paroi est la résultante de tous ces flux :

$$
\begin{aligned}
\varphi_{\mathrm{p}}=-\alpha_{\mathrm{s}} \varphi_{\mathrm{s}}+h_{r}\left(T_{\mathrm{p}}-T_{\mathrm{r}}\right)+h_{\mathrm{c}}( & \left.T_{\mathrm{p}}-T_{\mathrm{a}}\right)+ \\
& +h_{\mathrm{v}}\left(T_{\mathrm{p}}-T_{\mathrm{a}}\right)+\varphi_{\mathrm{v}} .
\end{aligned}
$$

Ce qui peut s'écrire encore sous une forme plus compacte :

$$
\varphi_{\mathrm{p}}=h\left(T_{\mathrm{p}}-T_{\mathrm{E}}\right)
$$

avec

$$
\begin{aligned}
h & =h_{r}+h_{\mathrm{c}}+h_{\mathrm{v}} \\
T_{\mathrm{E}} & =\frac{\left(h_{\mathrm{c}}+h_{\mathrm{v}}\right) T_{\mathrm{a}}+h_{r} T_{r}+\alpha_{\mathrm{s}} \varphi_{\mathrm{s}}-\varphi_{\mathrm{v}}}{h}
\end{aligned}
$$

$h$ est le coefficient global de transfert, $T_{\mathrm{E}}$ la température équivalente.

Déterminer le flux pariétal $\varphi_{\mathrm{p}}$ revient à connaître à chaque instant $T_{\mathrm{p}}, h$ et $T_{\mathrm{E}}$.

$h$ et $T_{\mathrm{E}}$ caractérisent donc globalement du point de vue énergétique, l'environnement microclimatique de la paroi à la température $T_{\mathrm{p}}$.

Cette analyse néglige le flux d'énergie lié à un transfert éventuel de masse à travers la paroi. Dans le cas d'une paroi avec revêtement hydrofuge (ce qui est le cas de la plupart des parois de construction actuelles) imperméable à l'eau liquide, mais perméable à la vapeur d'eau ce flux est négligeable (nul pour des parois isolées, avec pare vapeur, il reste inférieur à $10^{-2} \mathrm{~W} / \mathrm{m}^{2}$ pour une paroi sans pare vapeur, non isolée, d'épaisseur $15 \mathrm{~cm}$ ). Elle ne s'applique donc pas au cas de la paroi poreuse et sans revêtement hydrofuge de surface. Ce cas n'est pas considéré dans le cadre de cet article.

\section{Principe de la détermination dú flux pariétal. -} On place dans la paroi, affleurant juste sa surface, un capteur isolé thermiquement de cette paroi et présentant les mêmes caractéristiques superficielles que la paroi (même rugosité, mêmes propriétés radiatives...).

Ce capteur, de surface $S$, est muni d'un système de régulation thermique, ou plus simplement, d'un système de chauffage.

3.1 CAPTEUR « ÉQUILIBRÉ ». - Si on asservit la puissance thermique $P$ délivrée par le système de régulation de telle manière que la température du capteur $T_{\mathrm{c}}$ soit à chaque instant égale à la température $T_{\mathrm{p}}$ de la paroi, alors le flux qui quitte le capteur est le même que celui qui quitte la paroi. Si le capteur est parfaitement isolé, on a en régime stationnaire

$$
\varphi_{\mathrm{d}}=\frac{P}{S}=\varphi_{\mathrm{p}}=h\left(T_{\mathrm{p}}-T_{\mathrm{E}}\right) .
$$

La réalisation d'un tel capteur équilibré est délicate car en raison des fluctuations microclimatiques, il faut prévoir pour la régulation, un double système de chauffage et de refroidissement. Nous avons préféré 


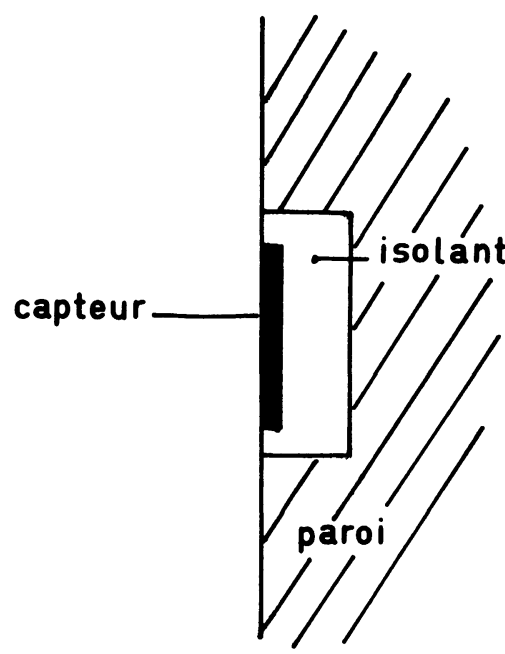

Fig. 1. - Position du capteur dans la paroi.

[Captor position in a wall.]

ne pas réaliser cet équilibrage strict et nous contenter de lois de chauffage imposées.

3.2 Capteur avec loi de Chauffage imposée. Ce type de capteur est seulement muni d'un système de chauffage programmable (flux nul, flux constant, impulsion, train d'impulsions...). La température du capteur $T_{\mathrm{c}}$ n'est donc pas égale à $T_{\mathrm{p}}$ (l'amplitude des lois de chauffage est choisie cependant de manière à ce que l'écart $T_{\mathrm{c}}-T_{\mathrm{p}}$ reste de l'ordre de 1 à $2^{\circ} \mathrm{C}$ ).

La température du capteur dépend des échanges avec l'environnement extérieur mais aussi de la température $T_{\mathrm{p}}$ de la paroi voisine. On peut écrire le flux $\varphi_{\mathrm{c}} \neq \varphi_{\mathrm{p}}$ qui quitte le capteur sous la forme :

$$
\varphi_{\mathrm{d}}=\varphi_{\mathrm{p}}+\delta \varphi
$$

$\delta \varphi$ est la perturbation de flux introduite par le chauffage non ajusté. On peut montrer que $\delta \varphi$ peut se mettre sous la forme [3] :

$$
\delta \varphi=h_{0}\left(T_{\mathrm{c}}-T_{\mathrm{p}}\right) .
$$

Expression dans laquelle $h_{0}$ est un coefficient global de transfert analogue à $h$ mais de valeur différente :

$$
h_{0}=h_{0 \mathrm{r}}+h_{0 \mathrm{c}}+h_{0 \mathrm{v}} \text {. }
$$

$h_{0_{\mathrm{r}}}$ est pratiquement identique à $h_{\mathrm{r}}$, par contre $h_{0 \mathrm{c}}$ ou $h_{0 v}$ sont des coefficients de convection locaux thermique ou massique et qui correspondent au développement d'une couche limite thermique ou massique liée à cette perturbation de flux $\delta \varphi$. S'agissant d'un début de couche limite, ils sont supérieurs à $h_{\mathrm{c}}$ et $h_{\mathrm{v}}$ et leur valeur dépend de la dimension du capteur (Fig. 2). Plus cette dimension sera petite dans le sens de l'écoulement, plus ces valeurs seront grandes (début de couche limite). Nous reviendrons sur ce point à propos de l'inertie du capteur au paragraphe 3.3 .

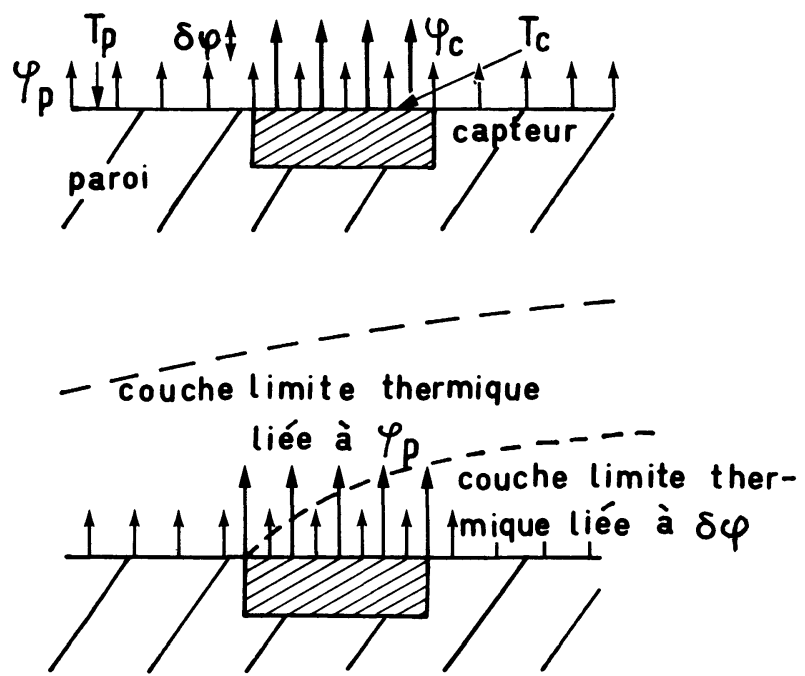

Fig. 2, - Perturbation thermique introduite par le capteur.

[Thermal disturbance caused by the captor.]

3.3 BILAN ÉNERGÉTIQUE SIMPLIFIÉ. - Dans un but de clarté, nous supposerons que le capteur est isotherme et parfaitement isolé de la paroi (une théorie plus exacte tenant compte d'une légère anisothermie, et d'une isolation imparfaite a été élaborée, nous en donnerons les résultats essentiels; nous verrons qu'elle n'apporte que des corrections mineures à la théorie simplifiée que nous allons présenter ici et ne change rien à son principe).

Si l'on désigne par $C$ la capacité calorifique du capteur rapportée à l'unité de surface, $T_{\mathrm{c}}$ sa température, $\varphi_{\mathrm{d}}$ la densité de flux dissipée par le système de chauffage, le bilan d'énergie conduit à la relation :

$$
\varphi_{\mathrm{d}}=h\left(T_{\mathrm{p}}-T_{\mathrm{E}}\right)+h_{0}\left(T_{\mathrm{c}}-T_{\mathrm{p}}\right)+C \frac{\mathrm{d} T_{\mathrm{c}}}{\mathrm{d} t} .
$$

Nous allons considérer différents types de lois de chauffage $\varphi_{\mathrm{d}}(t)$.

- Capteur inerte : il s'agit du capteur pour lequel $\varphi_{\mathrm{d}}=0$. Dans ce cas, la température du capteur que nous noterons $T_{\mathrm{c} 0}$ varie uniquement en raison des fluctuations microclimatiques ou des variations de $T_{\mathrm{p}}$. On a la relation :

$$
h\left(T_{\mathrm{p}}-T_{\mathrm{E}}\right)+h_{0}\left(T_{\mathrm{c} 0}-T_{\mathrm{p}}\right)+C \frac{\mathrm{d} T_{\mathrm{c} 0}}{\mathrm{~d} t}=0
$$

d'où l'on déduit :

$\varphi_{\mathrm{p}}=h\left(T_{\mathrm{p}}-T_{\mathrm{E}}\right)=h_{0}\left(T_{\mathrm{p}}-T_{\mathrm{c} 0}\right)-C \frac{\mathrm{d} T_{\mathrm{c} 0}}{\mathrm{~d} t}$.

Si le terme $C \frac{\mathrm{d} T_{\mathrm{c} 0}}{\mathrm{~d} t}$ est négligeable $(C$ petit et périodes des fluctuations grandes devant $C / h_{0}$ ), on 
a alors la relation plus simple :

$$
\varphi_{\mathrm{p}}=h\left(T_{\mathrm{p}}-T_{\mathrm{E}}\right) \simeq h_{0}\left(T_{\mathrm{p}}-T_{\mathrm{c} 0}\right) .
$$

Ainsi, à chaque instant les transferts superficiels paroi-environnement peuvent être caractérisés par $T_{\mathrm{p}}$ et l'un des deux couples $\left(h, T_{\mathrm{E}}\right)$ ou $\left(h_{0}, T_{\mathrm{c} 0}\right)$. Nous allons voir que $h_{0}$ et $T_{\mathrm{c} 0}$ sont beaucoup plus faciles à mesurer que $h$ et $T_{\mathrm{E}}$.

- Capteur avec loi de chauffage $\varphi_{\mathrm{d}}(t) \neq 0$. Constante de temps du capteur : l'équation énergétique (9) peut être simplifiée en lui retranchant l'équation (10).

En posant $\theta(t)=T_{\mathrm{c}}(t)-T_{\mathrm{c} 0}(t)$ il vient :

$$
\varphi_{\mathrm{d}}=h_{0} \theta+C \frac{\mathrm{d} \theta}{\mathrm{d} t} .
$$

L'enregistrement de la loi $\theta(t)$ pour une loi $\varphi_{\mathrm{d}}$ donnée permet de trouver $h_{0}$ à partir de (12). On détermine ensuite le flux pariétal $\varphi_{\mathrm{p}}$ à partir des enregistrements de $T_{\mathrm{p}}$ et $T_{\mathrm{c} 0}$ et des relations (10) ou (11). Bien entendu, on a intérêt à réaliser des capteurs dont l'inertie thermique est la plus faible possible de manière à utiliser la relation (11). Afin de préciser ce point, considérons le cas d'une loi de chauffage constante $\varphi_{\mathrm{d}}=\varphi_{0}$ avec comme condition initiale $\theta=0$ pour $t=0$.

La solution de (12) est

$$
\theta=\frac{\varphi_{0}}{h_{0}}\left(1-\exp \left[-\frac{h_{0}}{C} t\right]\right)
$$

On voit que la constante de temps du capteur est $\tau=C / h_{0}$.

En pratique pour $t>3 \tau$, le capteur aura atteint son équilibre $\theta_{\infty}=\varphi_{0} / h_{0}$.

Si l'on veut un capteur très peu inerte, il faudra donc que $C$ soit le plus petit possible et que $h_{0}$ soit le plus grand possible. Or $h_{0}$ est la somme d'un coefficient de rayonnement $h_{0 \mathrm{r}}$ et de 2 coefficients de convection $h_{0 \mathrm{c}}$ et $h_{0 \mathrm{v}}$ qui seront d'autant plus grands que la dimension du capteur sera faible (début de couche limite). En choisissant cette dimension suffisamment petite, on pourra obtenir une inertie très faiblè. Pour une inertie thermique fixée (constante de temps $\left.\tau=C / h_{0}\right)$ le capteur suivra, sans introduire de retard notable, les fluctuations dont la période sera grande devant $\tau$.

Exemple de calcul de $h_{0}$ :

- Capteur alimenté à flux constant : on a $\varphi_{d}=\varphi_{0}$, la solution(13) montre que pour $t$ suffisamment grand, la mesure de $\theta_{\infty}$ et de $\varphi_{0}$ donne $h_{0}$.

- Capteur avec loi de flux impulsionnelle : on a

$$
\begin{array}{llll}
\varphi_{\mathrm{d}}=\varphi_{0} & \text { pour } & 0<t<t_{0} \\
\varphi_{\mathrm{d}}=0 & \text { pour } & t>t_{0}
\end{array}
$$

on suppose qu'à l'état initial $t=0, \theta=0$ et que

$$
t_{0} \ll C / h_{0} \text {. }
$$

La solution de (12) est :

$$
\begin{aligned}
& \theta=\frac{\varphi_{0}}{h_{0}}\left(1-\exp \left[-\frac{h_{0}}{C}\right] t\right) \sim \frac{\varphi_{0}}{C} t \\
& \text { pour } 0<t<t_{0} \\
& \theta=\theta_{\mathrm{M}} \exp \left[\frac{-h_{0}}{C}\left(t-t_{0}\right)\right] \text { pour } t>t_{0}
\end{aligned}
$$

$\theta_{\mathrm{M}}=\frac{\varphi_{0} t_{0}}{C}$ est la température maximale atteinte pour $t=t_{0}$ (Fig. 3).

Seule la branche correspondant au refroidissement dépend de $h_{0}$. En mesurant la pente $m$ de $\operatorname{Ln} \theta / \theta_{\mathrm{M}}$ en fonction de $t-t_{0}$, on en déduit la constante de temps du capteur

$$
\tau=\frac{-1}{m} \text { et } h_{0}=-m C .
$$

- Capteur avec loi de flux périodique $\tau_{\mathbf{i}}$ (train d'impulsions) : Si la période $\tau_{\mathrm{i}}$ du train d'impulsion est grande devant $\tau$, chaque impulsion peut être considérée séparément et on est ramené au cas précédent. Par contre si $\tau_{\mathrm{i}}$ est du même ordre que $\tau$ le capteur n'a pas le temps de se refroidir complètement entre deux impulsions successives, on atteint un régime périodique établi dans lequel la température du capteur oscille entre deux valeurs $\theta_{\mathrm{M}}$ et $\theta_{\mathrm{m}}$.

L'enregistrement de $\theta=T_{\mathrm{c}}-T_{\mathrm{c} 0}$ permet de calculer encore $h_{0}$ et $T_{\mathrm{c} 0}[4,5]$.

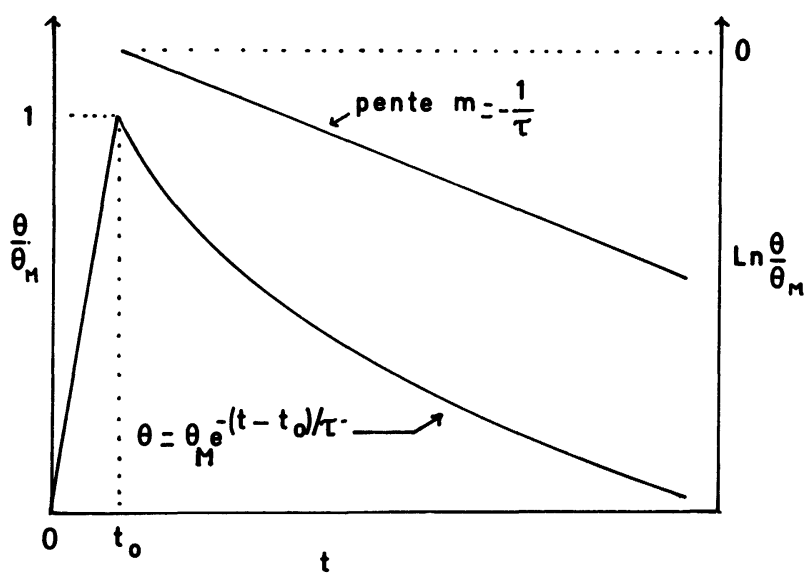

Fig. 3. - Variation de la température du capteur avec impulsion de flux. Théorie simplifiée : capteur isotherme.

[Evolution of captor temperature with an impulse flux simplified theory : isothermal captor.]

4. Description des capteurs et du dispositif d'essai. 4.1 DisPOSITIF D'ESSAI. - Le dispositif expérimental figure 4 qui a été réalisé comprend une veine d'expérience et les capteurs. La veine est à section rectangulaire et l'une de ses faces est constituée par une 


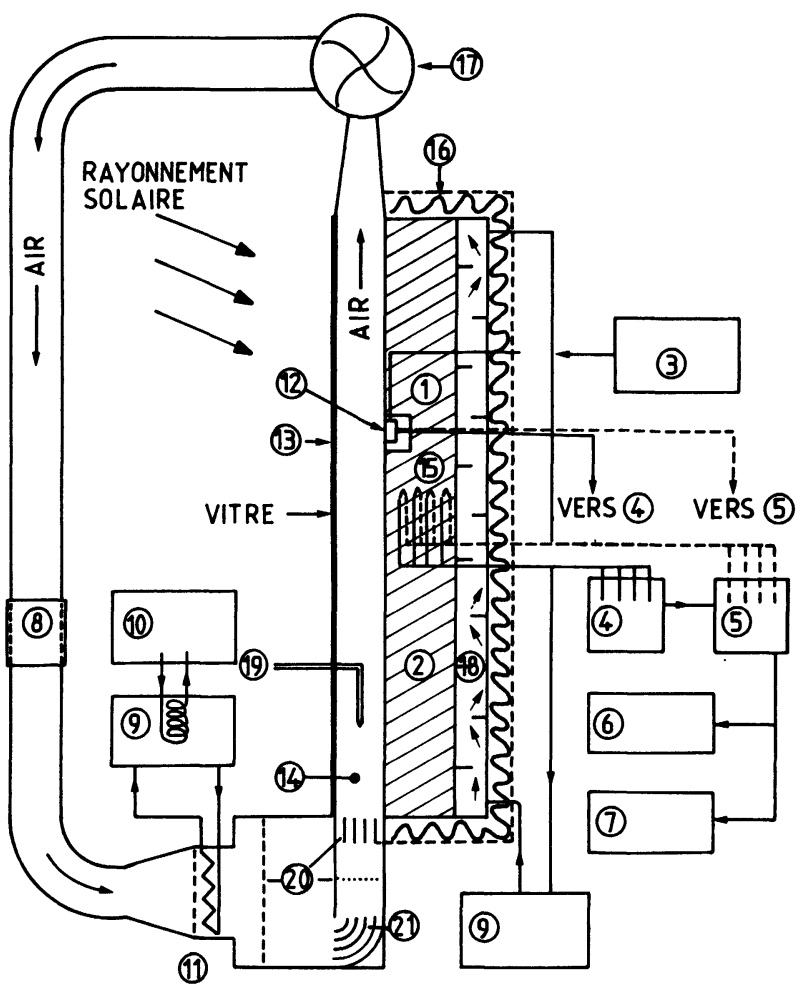

Fig. 4. - Schéma du dispositif expérimental : 1. Capteur. 2. Paroi de plâtre. 3. Système de chauffage. 4. Boîte froide. 5. Boîte de dérivation. 6. Microvolmètre. 7. Enregistreur. 8. Débitmètre. 9. Thermostat à circulation. 10. Cryostat. 11. Echangeur. 12. Thermocouple situé dans le capteur. 13. Thermocouple situé sur la vitre. 14. Thermocouple situé dans l'air. 15. Thermocouples situés dans la paroi. 16. Isolant. 17. Ventilateur. 18. Source thermique (circulation d'eau). 19. Prise de pression. 20. Grilles, et nid d'abeille. 21. Déflecteurs.

[Experimental device.]

paroi de plâtre, d'épaisseur $7 \mathrm{~cm}$ sur laquelle sont implantés les capteurs. La face opposée de cette veine est transparente au rayonnement visible et opaque aux infrarouges, la paroi de plâtre est balayée par un écoulement d'air à vitesse et température réglable (de 0 à $8 \mathrm{~m} / \mathrm{s}$ pour la vitesse et de 7 à $30^{\circ} \mathrm{C}$ pour la température). Grâce à un ensemble de grilles placées en amont le régime d'écoulement sur la plaque est turbulent. L'épaisseur de la veine a été calculée de façon à ce que l'écoulement soit comparable à un écoulement sur plaque plane (avec toutefois un léger gradient de pression); les couches thermiques se développant sur la paroi et la vitre ne se rejoignent pas dans le domaine de vitesses étudiées. L'autre face de la paroi en plâtre est en contact avec une source thermique à niveau réglable, ce qui permet de faire varier le flux thermique traversant cette plaque de plâtre.

Des thermocouples disposés dans l'écoulement, sur la face interne de la vitre et selon l'épaisseur de la paroi permettent de déterminer la température de l'air $T_{a}$, la température radiative $T_{\mathrm{r}}$ de la vitre, la température de la paroi $T_{\mathrm{p}}$ et le gradient de température au sein de la paroi $\mathrm{d} T_{\mathrm{p}} / \mathrm{d} x$.

On en déduit en régime permanent et pour le montage considéré

- le flux pariétal

$$
\varphi_{\mathrm{p}}=-\lambda_{\mathrm{p}} \frac{\mathrm{d} T_{\mathrm{p}}}{\mathrm{d} x}
$$

$\lambda_{\mathrm{p}}$ conductivité de la paroi

- le flux radiatif de grandes longueurs d'ondes

$$
\varphi_{\mathrm{r}}=\frac{\sigma\left(T_{\mathrm{p}}^{4}-T_{\mathrm{r}}^{4}\right)}{\frac{1}{\varepsilon_{\mathrm{p}}}+\frac{1}{\varepsilon_{\mathrm{v}}}-1}
$$

$\varepsilon_{\mathrm{p}} \varepsilon_{\mathrm{v}}$ sont respectivement les émissivités de la paroi de plâtre et de la vitre; $\sigma$ est la constante de Stefan Bolzman

- et le coefficient de rayonnement

$$
h_{\mathrm{r}}=\frac{\varphi_{\mathrm{r}}}{T_{\mathrm{p}}-T_{\mathrm{r}}}
$$

- le flux convectif $\varphi_{\mathrm{c}}=\varphi_{\mathrm{p}}-\varphi_{\mathrm{r}}$, et le coefficient de convection

$$
h_{\mathrm{c}}=\frac{\varphi_{\mathrm{c}}}{T_{\mathrm{p}}-T_{\mathrm{a}}}
$$

- enfin le coefficient global de transfert $h=h_{\mathrm{c}}+h_{\mathrm{r}}$ et la température équivalente $T_{\mathrm{E}}=T_{\mathrm{p}}-\frac{\varphi_{\mathrm{p}}}{h}$.

4.2 Capteur. - Afin d'obtenir la plus faible capacité thermique possible (cf. § 3.4) le capteur qui a été réalisé, est constitué (Fig. $5 a$ ) d'une pastille circulaire comprenant une couche de $3 / 100 \mathrm{~mm}$ de cuivre déposé sur un support en bakélite $(1,35 \mathrm{~mm})$ et un élément chauffant collé sur l'autre face. Un thermocouple chromel alumel $\left(41 \mu \mathrm{V} /{ }^{\circ} \mathrm{C}\right)$ nous donne la température de surface du capteur. Ce thermocouple est du type à conducteurs séparés, c'est-à-dire que les fils chromel et alumel sont soudés séparément sur la feuille de cuivre (Fig. $5 b$ ), la jonction thermoélectrique étant assurée par le cuivre lui-même.

Le capteur est isolé de la paroi étudiée par un manchon en polyuréthane, afin que la quasi-totalité du flux thermique dissipé par l'élément chauffant soit évacué vers l'ambiance externe.

Afin de conserver les mêmes propriétés de surface que la paroi, le capteur est recouvert d'un enduit identique à celle-ci. Deux configurations différentes ont été étudiées pour ce manchon isolant. Dans la première (capteur A) la largeur $\delta r$ de l'espace annulaire entre le capteur et la paroi est faible $(\delta r=1 \mathrm{~mm})$ 
alors que dans la seconde (capteur B) pour éviter les fuites radiales trop importantes, cet espace a été porté à $5 \mathrm{~mm}$ et la dimension du capteur réduite d'autant.

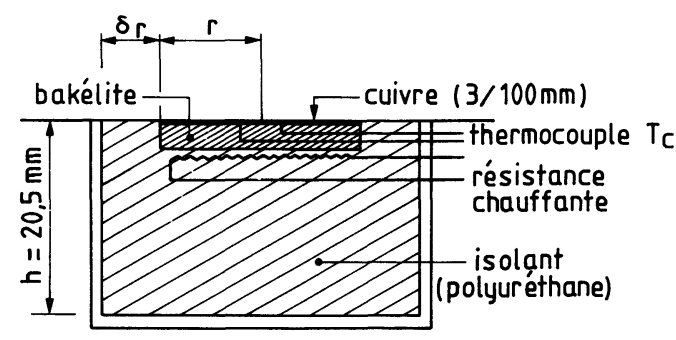

Fig. $5 a$. - Schéma du capteur : capteur A : $2 r=29,5 \mathrm{~mm}$; capteur B : $2 r=21 \mathrm{~mm}$.

[Sketch of captor : captor A : $2 r=29.5 \mathrm{~mm}$; captor B : $2 r=21 \mathrm{~mm}$.]

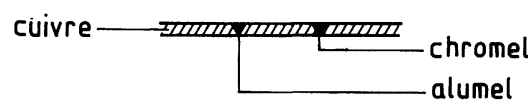

Fig. $5 b$. - Détail du thermocouple du capteur.

[Detail of the captor thermocouple.]

Des études par thermographie infrarouge (sensibilité $0,2^{\circ} \mathrm{C}$ ) n'ont pas permis de déceler dans la gamme de vitesse étudiée une non uniformité de la température de surface de chacun des capteurs.

5. Bilan énergétique tenant compte de l'isolation imparfaite du capteur. - Nous avons vu dans la théorie simplifiée que le flux de paroi pouvait être déduit de l'enregistrement de $T_{\mathrm{p}}$ de $T_{\mathrm{c} 0}$ et de $h_{0}$ par la relation (10). La théorie plus exacte prenant en compte l'isolation imparfaite de capteur et de la paroi, ainsi que l'influence de la couronne isolante qui l'entoure conduit à substituer à l'équation (12), l'équation (16)

$$
\varphi_{\mathrm{d}}=\left(h_{0}+k_{\mathrm{i}}\right) \theta+C \frac{\mathrm{d} \theta}{\mathrm{d} t}
$$

$k_{\mathrm{i}}$ représente un coefficient lié au transfert de chaleur parasite paroi capteur à travers l'isolant. Cette équation peut s'écrire sous une forme plus compacte.

$$
\varphi_{\mathrm{d}}=h_{0}^{\prime} \theta+C \frac{\mathrm{d} \theta}{\mathrm{d} t}
$$

avec

$$
h_{0}^{\prime}=h_{0}+k_{\mathrm{i}} .
$$

La théorie simplifiée élaborée en 3.3 (capteur inerte et capteur équilibré $T_{\mathrm{p}}=T_{\mathrm{c}}$ ) se transpose intégralement :

- $h_{0}$ étant remplacé par $h_{0}^{\prime}$

- $T_{\mathrm{c} 0}$ étant remplacé par $T_{\mathrm{c} 0}^{\prime}$ qui est la nouvelle température d'équilibre du capteur non chauffé mais imparfaitement isolé.
Les valeurs directement mesurables sont $h_{0}^{\prime}$ et $T_{\mathrm{c} 0}^{\prime}$ L'équation d'équilibre du capteur imparfaitement isolé montre que le flux pariétal $\varphi_{\mathrm{p}}$ se déduit directement du couple $h_{0}^{\prime}, T_{\mathrm{c} 0}^{\prime}$ par la relation :

$$
\varphi_{\mathrm{p}}=\frac{h_{0}^{\prime}}{k}\left(T_{\mathrm{p}}-T_{\mathrm{c} 0}^{\prime}\right)
$$

où $k$ est une caractéristique du capteur qui dépend du rapport des conductivités $\lambda_{i}$ et $\lambda_{p}$ de l'isolant et de la paroi, et d'un facteur géométrique $f(r, \delta r)$ lié à l'épaisseur de l'espace annulaire isolant et au rayon $r$ du capteur. On a

$$
k=f(r, \delta r)\left(1-\frac{\lambda_{\mathrm{i}}}{\lambda_{\mathrm{p}}}\right)
$$

Les constantes $k$ et $k_{\mathrm{i}}$ ont été déterminées expérimentalement pour les deux types de capteurs. On a trouvé respectivement :

$$
\begin{array}{lll}
\text { capteur A } & k=1,12 & k_{\mathrm{i}}=11,32 \\
\text { capteur B } & k=1,5 & k_{\mathrm{i}}=3,22 .
\end{array}
$$

6. Résultats. - -6.1 RÉPONSE À DES VARIATIONS DE VITESSES D'AIR. DÉTERMINATION DE $h_{0}^{\prime}$. - L'expérience confirme la sensibilité du capteur à la vitesse de l'air (Fig. 6).

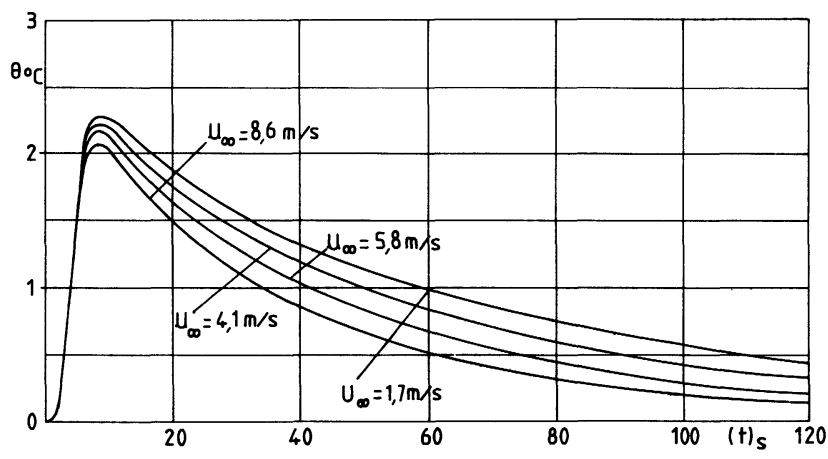

Fig. 6. - Variation de la température du capteur B, soumis à une impulsion de flux de durée $t_{0}=4 \mathrm{~s}$, en fonction de la vitesse de l'air.

[Variation of the captor B temperature, versus air velocity (with an impulse flux during $t_{0}=4 \mathrm{~s}$ ).]

Les figures 7 et 8 donnent pour chaque type de capteur, l'évolution $\operatorname{Ln} \theta / \theta_{\mathrm{M}}$ en fonction de $\left(t-t_{0}\right)$ pour deux valeurs extrêmes de $h_{0}^{\prime}$.

On constate que, excepté le début du refroidissement, la loi est bien exponentielle. 


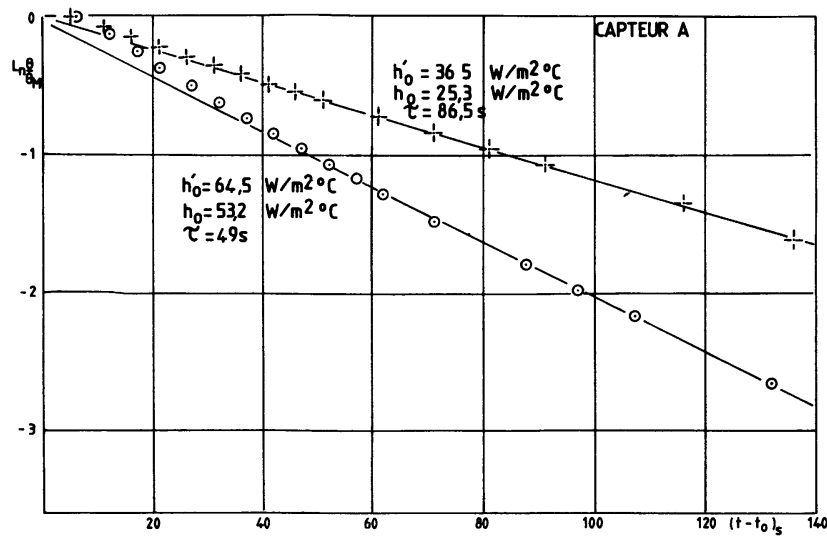

Fig. 7. - Décroissance de la température du capteur A après une impulsion de flux de durée $t_{0}=4 \mathrm{~s}:-,-, \odot$ : décroissance expérimentale; — décroissance théorique (capteur isotherme).

[Decreasing of the captor A temperature, after an impulse flux during $t_{0}=4 \mathrm{~s}:-\div-\odot:$ Experimental decreasing; theoretical decreasing (isothermal captor).]

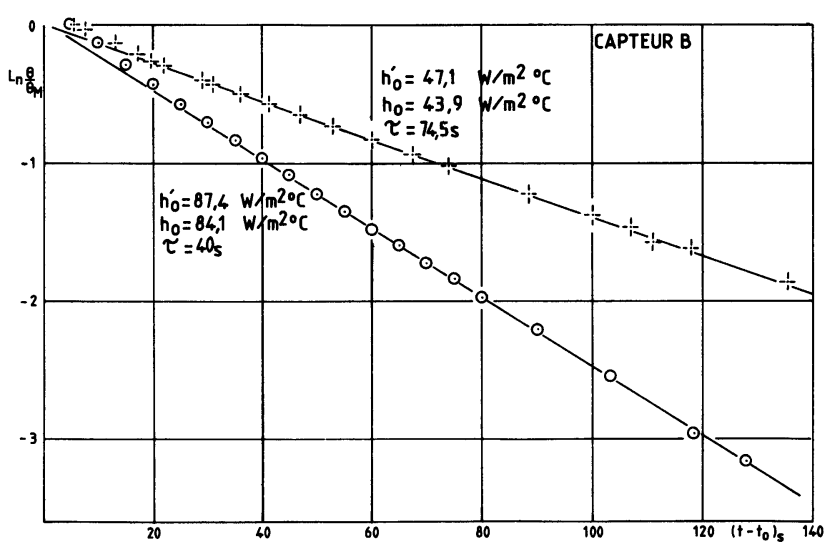

Fig. 8. - Décroissance de la température du capteur B après une impulsion de flux de durée $t_{0}=4 \mathrm{~s}$. $-\odot$ : décroissance expérimentale; - décroissance théorique (capteur isotherme).

[Decreasing of the captor B temperature, after an impulse flux during $t_{0}=4 \mathrm{~s}:-\div-\odot:$ experimental decreasing; - theoretical decreasing (isothermal captor).]

Les figures 9 et 10 représentent les courbes expérimentales et la théorie simplifiée conforme à la figure 3. L'écart entre les deux, essentiellement aux faibles valeurs du temps est dû aux effets de conduction selon l'épaisseur du capteur qui ne peut être considéré tout à fait isotherme comme le suppose la théorie précédente. Ces effets sont responsables du retard à l'échauffement au début $\left(t<t_{0}\right)$ et du maximum arrondi; la solution plus exacte s'exprime sous forme d'une différence de deux séries de Fourrier. Au bout d'un

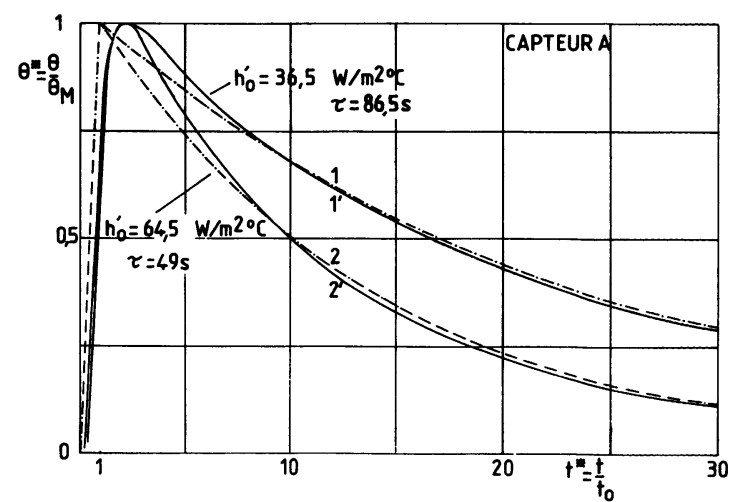

Fig. 9. - Variation de la température du capteur A, soumis à une impulsion de flux de durée $t_{0}=4 \mathrm{~s}$. - Variation expérimentale $\left(1^{\prime}\right.$ et $\left.2^{\prime}\right)$. $-\cdot-\cdot-$ Variation théorique (capteur isotherme) $(1$ et 2$) \tau^{*}=\frac{\tau}{t_{0}}$

$$
\begin{array}{ll}
0 \leqslant t^{*} \leqslant 1 & \theta^{*}=t^{*} \\
1 \leqslant t^{*}<\infty & \theta^{*}=\exp \left[-\left(\frac{t^{*}-1}{\tau^{*}}\right)\right]
\end{array}
$$

[Variation of the captor A temperature (with an impulse flux during $t_{0}=4 \mathrm{~s}$ ) : - Experimental graphs $1^{\prime}$ and $2^{\prime}$ ). -. - Theoretical (isothermal capteur) graphs 1 and 2.]

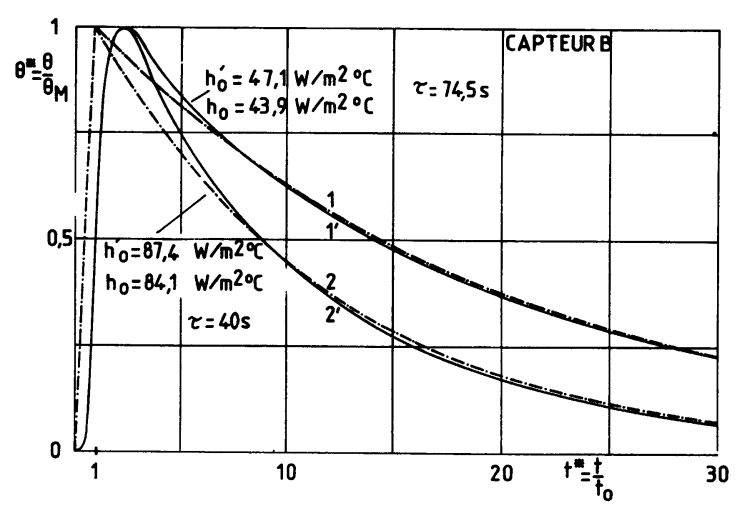

Fig. 10. - Variation de la température du capteur B, soumis à une impulsion de flux de durée $t_{0}=4 \mathrm{~s}$. - Variation expérimentale $\left(1^{\prime}\right.$ et 2$),-\cdot-\cdot-$ Variation théorique (capteur isotherme) (1 et 2) $\tau^{*}=\frac{\tau}{t_{0}}$

$$
\begin{array}{ll}
0 \leqslant t^{*} \leqslant 1 & \theta^{*}=t^{*} \\
1 \leqslant t^{*}<\infty & \theta^{*}=\exp \left[-\left(\frac{t^{*}-1}{\tau^{*}}\right)\right] .
\end{array}
$$

[Variation of the captor B temperature (with an impulse flow during $t_{0}=4 \mathrm{~s}$ ): - Experimental (graphs 1' and 2'). - - - Theoretical (isothermal capteur) graphs 1 and 2.]

temps suffisamment grand la solution peut être limitée à son premier terme qui est de la forme :

$$
A_{1} f_{1}(l)\left[\exp \left(\frac{a u_{1}^{2} t_{0}}{l^{2}}\right)-1\right] \exp \left(-\frac{a u_{1}^{2} t}{l^{2}}\right)
$$


Tableau I. - Mesures de $h_{0}^{\prime}$ pour le capteur $A$

\begin{tabular}{|c|c|c|c|c|c|c|}
\hline & $u_{\infty} \mathrm{ms}^{-1}$ & 1,53 & 2,35 & 3,45 & 4,74 & 5,81 \\
\hline & $\tau \quad \bar{s}$ & $\overline{86,5}$ & $\overline{65}$ & $\overline{58}$ & $\overline{52}$ & 49 \\
\hline \multirow{2}{*}{ Mesures de $h_{0}^{\prime}$ en transitoire $\left(\mathrm{W} / \mathrm{m}^{2}{ }^{\circ} \mathrm{C}\right)$} & $h_{0}^{\prime}=\frac{C}{\tau}$ & 36,5 & 46,5 & 54,5 & 60,8 & 64,5 \\
\hline & $h_{0}^{\prime}=\frac{\lambda}{l} u \operatorname{tg} u$ & 35,9 & 46,6 & 55,5 & 62,7 & 67,04 \\
\hline Mesures de $h_{0}^{\prime}$ en permanent $\left(\mathrm{W} / \mathrm{m}^{2}{ }^{\circ} \mathrm{C}\right)$ & $h_{0}^{\prime}=\frac{\Delta \varphi}{\Delta T_{\mathrm{c}}^{\infty}}$ & 36,3 & 47,3 & 55 & 59,3 & 67,5 \\
\hline
\end{tabular}

Tableau II. - Mesures de $h_{0}^{\prime}$ pour le capteur B

\begin{tabular}{|c|c|c|c|c|c|c|c|}
\hline & $u_{\infty} \mathrm{ms}^{-1}$ & $\frac{1,72}{-}$ & 3,25 & $\frac{4,06}{-}$ & $\frac{5,15}{-}$ & 5,84 & $\frac{8,60}{-}$ \\
\hline & $\tau \quad \mathrm{s}$ & 74,4 & 59,9 & 56,8 & 52,1 & 45,2 & 40,1 \\
\hline \multirow{2}{*}{ Mesures en transitoire $\left(W / \mathrm{m}^{2}{ }^{\circ} \mathrm{C}\right)$} & $h_{0}^{\prime}=\frac{C}{\tau}$ & 47,1 & 58,5 & 61,6 & 67,2 & 77,4 & 87,4 \\
\hline & $h_{0}^{\prime}=\frac{\lambda}{l} u \operatorname{tg} u$ & 45,7 & 58 & 61,7 & 68,1 & 80,3 & 92,7 \\
\hline Mesures en permanent & $h_{0}^{\prime}=\frac{\Delta \varphi}{\Delta T_{\mathrm{c}}^{\infty}}$ & 47,5 & 58 & 61 & 68,8 & 77,2 & 88,1 \\
\hline
\end{tabular}

$l$ est l'épaisseur du capteur, $a$ sa diffusivité thermique et $u_{1}$ la première racine de l'équation transcendante :

$$
u \operatorname{tg} u=\frac{h_{0}^{\prime} l}{\lambda_{\mathrm{b}}}
$$

$\lambda_{b}$ conductivité du matériau constituant le capteur.

On retrouve donc une loi exponentielle du même type que celle que donne la solution capteur isotherme, la constante de $\tau=l^{2} / a u_{1}^{2}$ est cependant légèrement plus grande que celle $\tau=C / h_{0}^{\prime}$ du capteur isotherme (pour $\frac{h_{0}^{\prime} l}{\lambda_{\mathrm{b}}} \simeq 0,01$ ces valeurs sont pratiquement identiques).

Les tableaux I et II donnent pour chaque capteur les valeurs de $h_{0}^{\prime}$ déduites soit de la formule approchée $\tau=\frac{C}{h_{0}^{\prime}}$, soit à partir des formules exactes :

$$
\tau=\frac{l^{2}}{a u_{1}^{2}} \quad \text { et } \quad h_{0}^{\prime}=\frac{\lambda_{\mathrm{b}}}{l} u_{1} \operatorname{tg} u_{1}
$$

On constate que ces valeurs sont très voisines sauf pour les faibles valeurs de $\tau$ où l'hypothèse d' isothermie est un peu moins justifiée. Par ailleurs on constate qu'à vitesse égale, le capteur $\mathrm{B}$, de plus petit diamètre que $\mathrm{A}$, a une inertie plus faible ( $h_{0}^{\prime}$ plus grand).

Afin de vérifier que ces valeurs de $h_{0}^{\prime}$ sont bien significatives nous avons voulu les comparer à des mesures directes de $h_{0}^{\prime}$ effectuées en régime permanent. Le principe de la mesure est le suivant : on note pour différents flux électriques $\varphi_{\mathfrak{d}}$ dissipés dans le capteur la température d'équilibre $T_{\mathrm{c}}^{\infty}$ de celui-ci, la pente de la droite $\varphi_{\mathrm{d}}=f\left(T_{\mathrm{c}}^{\infty}\right)$ doit être égale à $h_{0}^{\prime}$ (Fig. 11).

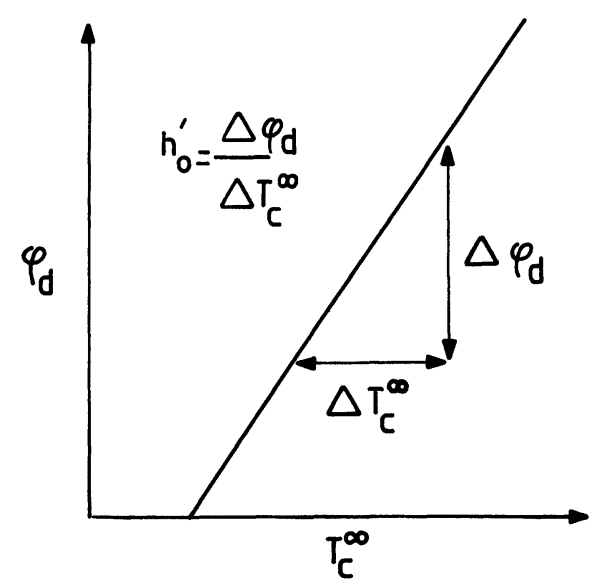

Fig. 11. - Principe de la mesure du coefficient $h_{0}^{\prime}$ en régime permanent.

[Determination of the factor $h_{0}^{\prime}$ is steady state.]

Les valeurs ainsi calculées sont également données dans les tableaux I et II. On constate que l'accord entre mesures en régime transitoire et mesures en régime permanent est très satisfaisant (l'écart est inférieur à $5 \%$ ).

6.2 Calcul du fluX Parítal $\varphi_{\mathrm{p}}$ - - La réponse transitoire du capteur permet de connaître $h_{0}^{\prime}$ et $T_{\mathrm{c} 0}^{\prime}$ et de calculer le flux pariétal par la relation (19) à condition de connaître $T_{\mathrm{p}}$. 
Tableau III $\left(h_{0}^{\prime}=\frac{\lambda_{\mathrm{b}}}{l} u \operatorname{tg} u\right)$

Capteur A

$u_{\infty}\left(\mathrm{ms}^{-1}\right)$

1,53

35,9

$h_{0}^{\prime}\left(\mathrm{Wm}^{-2} \mathrm{~K}^{-1}\right)$

$T_{\mathrm{p}}\left({ }^{\circ} \mathrm{C}\right)$

$T_{\mathrm{c} 0}^{\prime}\left({ }^{\circ} \mathrm{C}\right)$

$\varphi_{\mathrm{pc}}\left(\mathrm{Wm}^{-2}\right)$

$\varphi_{\mathrm{pg}}\left(\mathrm{Wm}^{-2}\right)$

$\frac{\varphi_{\mathrm{pc}}-\varphi_{\mathrm{pg}}}{\varphi_{\mathrm{pg}}}(\%)$

\section{9,6}

28,3

40,7

43,9

$-7 \%$
2,35

46,6

26,8

25,3

64,9

57,4

$+13 \%$
3,45

55,5

24,2

23.4

42,3

42,2

$-0,3 \%$
4,7

62,7

23,3

22,4

50,5

47

$+8 \%$
5,81

67,04

24,9

24,3

38,0

39,5

$-4 \%$

Tableau IV. $-\left(h_{0}^{\prime}=\frac{\lambda_{\mathrm{b}}}{l} u \operatorname{tg} u\right)$

\section{Capteur B}

$u_{\infty}\left(\mathrm{ms}^{-1}\right)$

$h_{0}^{\prime}\left(\mathrm{Wm}^{-2} \mathrm{~K}^{-1}\right)$

$T_{\mathrm{p}}\left({ }^{\circ} \mathrm{C}\right)$

$T_{\mathrm{c} 0}^{\prime}\left({ }^{\circ} \mathrm{C}\right)$

$\varphi_{\mathrm{pc}}\left(\mathrm{Wm}^{-2}\right)$

$\varphi_{\mathrm{pg}}\left(\mathrm{Wm}^{-2}\right)$

$\frac{\varphi_{\mathrm{pc}}-\varphi_{\mathrm{pg}}}{\varphi_{\mathrm{pg}}}(\%)$

\section{1,72}

45,7

20,2

18,9

38,9

39,3

$-1$

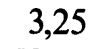

58

14,6

12,8

72,4

66,3

$+9$
Les tableaux III et IV donnent pour chaque type de capteur les flux $\varphi_{\mathrm{pc}}$ ainsi calculés, ainsi que ceux que l'on peut déduire directement de la mesure du gradient de température dans la paroi en régime permanent $\varphi_{\mathrm{pg}}=-\lambda_{\mathrm{p}} \frac{\mathrm{d} T_{\mathrm{p}}}{\mathrm{d} x} ;$ considérant l'aspect aléatoire des écarts on peut dire que la concordance est très correcte.

Les températures sont données dāns les tableaux au $1 / 10$ de degrés pour des raisons de présentation. En réalité nous estimons la précision des mesures de température de l'ordre du $1 / 20^{\mathrm{e}}$ degré (mesure au microvoltmètre des tensions délivrées par les thermocouples chromel Alumel $-41 \mu \mathrm{V} / \mathrm{K}$.

6.3 RELATIONS ENTRE LES COEFFICIENTS DE CONVECTION $h_{\mathrm{c}}$ ET $h_{0 \mathrm{c}}-6.3 .1$ Corrélations expérimentales. Les coefficients de transfert locaux $h_{0}^{\prime}$ liés à la perturbation de température $\delta T_{\mathrm{p}}$ différent des coefficients de transfert $h$ pour la paroi à $T_{\mathrm{p}}$ (voir $\left.\S 3.2\right)$. On a calculé les coefficients de convection en retranchant au coefficient global de transfert, le coefficient de rayonnement $h_{\mathrm{r}}$ et le coefficient de fuite $k_{\mathrm{i}}$. Les coefficients $h_{\mathrm{r}}$ sont de l'ordre de $5 \mathrm{~W} / \mathrm{m}^{2} \mathrm{~K}$, étant donné le domaine de température des parois et de la vitre; $k_{\mathrm{i}}$ est égal à 11,32 pour le capteur A et 3,22 pour le capteur B.

On a respectivement :

$$
\begin{aligned}
& h_{\mathrm{c}}=h-h_{\mathrm{r}} \\
& h_{0 \mathrm{c}}=h_{0}^{\prime}-h_{\mathrm{r}}-k_{\mathrm{i}} .
\end{aligned}
$$

Les figures 12 et 13 représentent l'évolution de $\log h_{\mathrm{c}}=f\left(\log h_{0 \mathrm{c}}\right)$ pour les deux types de capteur.

$\begin{array}{rccr}4,06 & 5,15 & 5,84 & 8,6 \\ 61,7 & 68,1 & 80,3 & 92,7 \\ 19,1 & 19 & 12,9 & 18,5 \\ 18,1 & 18,1 & 11,5 & 17,8 \\ 44,1 & 41,6 & 76,4 & 47,2 \\ 44,6 & 44,9 & 73,9 & 47,1 \\ -1,1 & -7 & +4 & +0,3\end{array}$

$\log h_{c}$

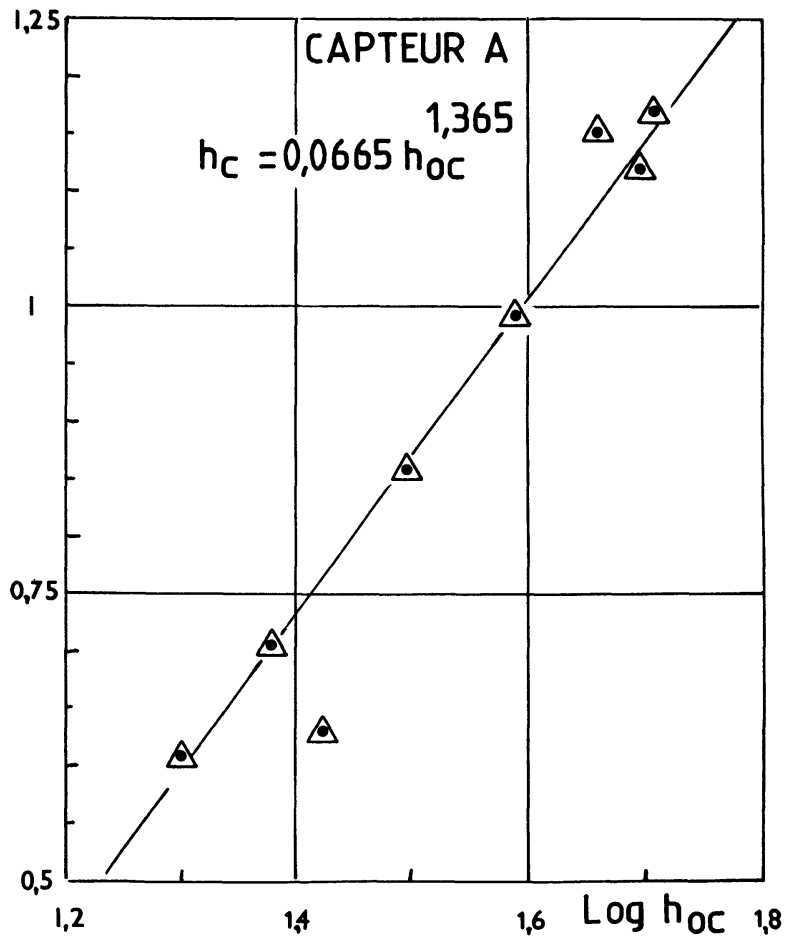

Fig. 12. - Corrélation expérimentale entre le coefficient de convection $h_{\mathrm{c}}$ lié à la paroi, et le coefficient de convection $h_{0 \mathrm{c}}$ lié au capteur (capteur A).

[Experimental correlation between the convection coefficient $h_{\mathrm{c}}$ referred to the wall and the convection coefficient $h_{0 \mathrm{c}}$ referred to the captor (captor A).] 


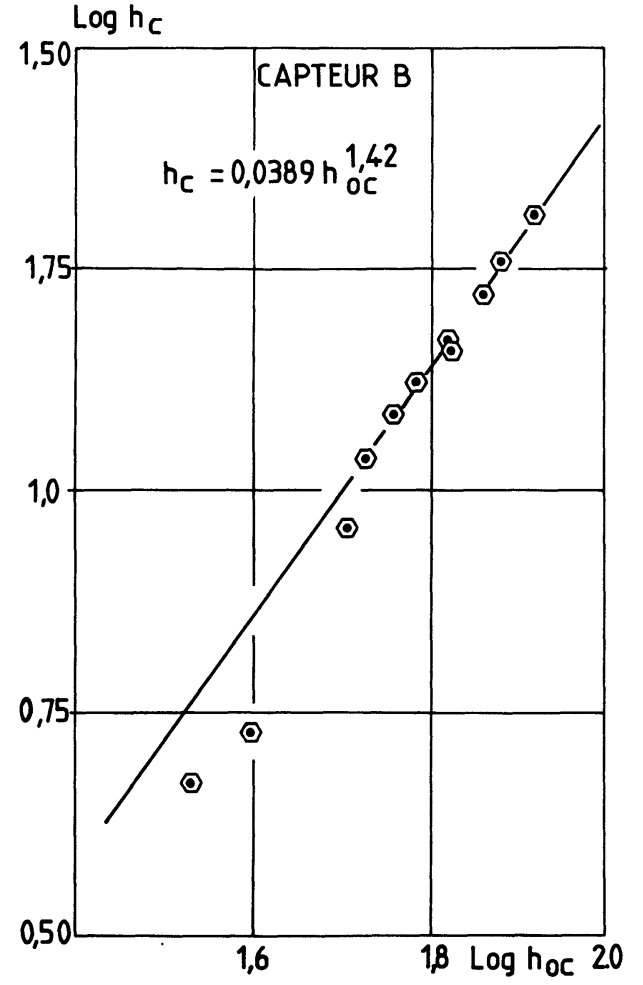

Fig. 13. - Corrélation expérimentale entre le coefficient de convection $h_{\mathrm{c}}$ lié à la paroi, et le coefficient de convection $h_{0 \mathrm{c}}$ lié au capteur (capteur B).

[Experimental correlation between the convection coefficient $h_{\mathrm{c}}$ referred to the wall and the convection coefficient $h_{0 \mathrm{c}}$ referred to the captor (captor B).]

Tableau V

\section{Capteur A}

Sans rayonnement solaire direct

$u_{\infty}\left(\mathrm{ms}^{-1}\right)$
${ }^{\circ} h\left(\mathrm{Wm}^{-2} \mathrm{~K}^{-1}\right)$
${ }^{*} h\left(\mathrm{Wm}^{-2} \mathrm{~K}^{-1}\right)$
${ }^{\circ} T_{\mathrm{E}}\left({ }^{\circ} \mathrm{C}\right)$
${ }^{*} T_{\mathrm{E}}\left({ }^{\circ} \mathrm{C}\right)$

$\begin{array}{cllll}1,53 & 2,35 & 3,45 & 4,74 & 5,8 \\ 9,6 & 12,6 & 16 & 19,9 & 21,8 \\ 8,9 & 12 & 14,9 & 18,5 & 19,2 \\ 25,0 & 22,3 & 21,6 & 20,9 & 23,1 \\ 25,0 & 21,4 & 21,4 & 20,4 & 22,9\end{array}$

On constate que les résultats sont bien corrélés par une loi de type puissance :

$$
h_{\mathrm{c}}=D h_{0 \mathrm{c}}^{n}
$$

avec $n=1,365$ et $D=0,0665$ pour le capteur $A$ figure $12 ; n=1,42$ et $D=0,0389$ pour le capteur B figure 13.

On en déduit donc une relation entre les coefficients globaux de transferts $h$ et $h_{0}^{\prime}$

$$
h=D\left(h_{0}^{\prime}-k_{\mathrm{i}}-h_{\mathrm{r}}\right)^{n}+h_{\mathrm{r}} \text {. }
$$

Dans le domaine de température de paroi et d'environnement considéré, $h_{\mathrm{r}}$ varie très peu et dans tous les cas peut être pris égal à $5 \mathrm{~W} / \mathrm{m}^{2} \mathrm{~K}$.

Il est donc possible à partir $h_{0}^{\prime}$ de déterminer le coefficient d'échange $h$ par la formule (24); la mesure de la température du capteur $T_{\mathrm{c} 0}^{\prime}$ et de la paroi $T_{\mathrm{p}}$, permet alors de connaître la température équivalente

$$
\begin{gathered}
T_{\mathrm{E}}=T_{\mathrm{p}}-\frac{\varphi_{\mathrm{p}}}{h} \\
T_{\mathrm{E}}=T_{\mathrm{p}}-\frac{h_{0}^{\prime}}{k} \cdot\left(T_{\mathrm{p}}-T_{\mathrm{c} 0}^{\prime}\right) \cdot \frac{1}{h}
\end{gathered}
$$

Les tableaux V et VI donnent les valeurs de $h$ et de $T_{\mathrm{E}}$ ainsi calculées par le capteur ainsi que celles que permet de déduire le dispositif expérimental par les mesures faites dans la paroi (cf. 4.1) en régime permanent.

Il convient de souligner qu'il s'agit des mêmes expériences que précédemment c'est-à-dire en l'absence de rayonnement solaire direct, ce qui n'exclut pas un certain rayonnement diffus susceptible de varier.

\section{Tableau VI}

\section{Capteur B}

$$
\begin{aligned}
& u_{\infty}\left(\mathrm{ms}^{-1}\right) \\
& { }^{\circ} h\left(\mathrm{Wm}^{-2} \mathrm{~K}^{-1}\right) \\
& { }^{*} h\left(\mathrm{Wm}^{-2} \mathrm{~K}^{-1}\right) \\
& { }^{\circ} T_{\mathrm{E}}\left({ }^{\circ} \mathrm{C}\right) \\
& { }^{*} T_{\mathrm{E}}\left({ }^{\circ} \mathrm{C}\right)
\end{aligned}
$$

Sans rayonnement solaire direct

$\begin{array}{llllrr}1,72 & 3,25 & 4,1 & 5,2 & 5,8 & 8,6 \\ 10,2 & 13,7 & 15,7 & 18,2 & 19,4 & 25,4 \\ 12,1 & 15 & 16,1 & 18 & 21,9 & 26,1 \\ 16,3 & 9,8 & 16,3 & 16,5 & 9,1 & 16,7 \\ 16,9 & 9,9 & 16,4 & 16,7 & 9,4 & 16,7\end{array}$

Avec rayonnement solaire direct

- Valeurs obtenues à partir du montage expérimental.

* : Valeurs obtenues à l'aide du capteur. 
Application : Mesure d'un flux solaire. - Nous venons d'analyser l'influence de la vitesse de l'air sur le comportement du capteur : cela se traduit par la variation de $h_{0}^{\prime}$. L'étude de l'influence du rayonnement solaire direct sur le capteur exige que les autres caractéristiques microclimatiques (vitesse d'air, température radiative $T_{\mathrm{r}} \ldots$ ) restent constantes. Cette condition n'est pas réalisée actuellement sur le montage à cause de la vitre destinée à canaliser l'air, qui absorbe en partie le rayonnement incident.

Les conditions n'ayant pas permis de mesures en rayonnement solaire, nous avons utilisé un rayonnement artificiel produit par des lampes, donc très riche en infrarouge. La vitre en absorbe la fraction de longueur d'onde supérieure à $2 \mu \mathrm{m}$, ce qui l'échauffe. Les résultats présentés (fig. 14) sont donc les effets des variations simultanées, du rayonnement $\varphi_{\mathrm{s}}$ de longueur d'onde inférieure à $2 \mu \mathrm{m}$ qui frappe le capteur, de la température radiative $T_{\mathrm{r}}$ et de la température de l'air.

Le capteur, comme la surface de paroi qui l'entoure, absorbe une fraction de ce flux incident $\varphi_{s}$, proportionnellement à son coefficient d'absorption $\alpha_{\mathrm{s}}$.

L'apparition brutale du rayonnement conduit donc donc à un échelon de flux absorbé ce qui peut se traduire plus simplement par l'augmentation instantanée de la température équivalente $T_{\mathrm{E}}$. La température de la paroi augmente ainsi que celle $d u$ capteur qui y est disposé.

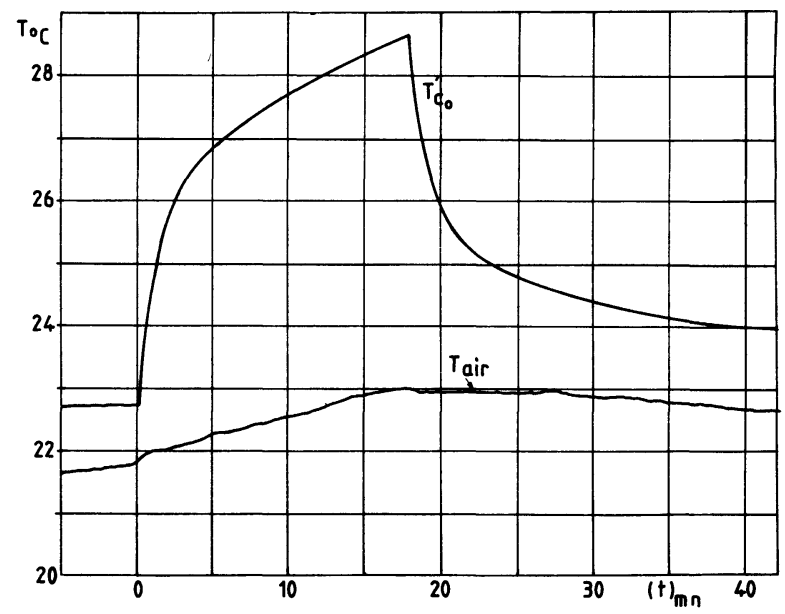

Fig. 14. - Réponse du capteur inerte à un rayonnement direct de durée égale à $18 \mathrm{~min}$.

[Inert captor reaction to a direct radiation during $18 \mathrm{~min}$.]

Le coefficient $h_{0}^{\prime}$ du capteur restant identique à ce qu'il était avant .l'apparition du rayonnement, le tableau V donne pour une valeur de $h_{0}^{\prime}$ la valeur de $h$ et $T_{E}$ en présence de rayonnement courte longueur d'onde. Les valeurs sont calculées à partir des données du capteur et de l'enregistrement de la température de paroi équations (25) et (24). Il n'est plus possible de déterminer les valeurs de $h$ et $T_{\mathrm{E}}$ à partir des mesures de températures en paroi $(\$ 4.1)$ car celle-ci n’est plus en régime permanent. Nous avons fait figurer entre parenthèses les valeurs de $T_{E}$ avant l'apparition du soleil. La variation de température équivalente $\Delta T_{\mathrm{E}}$ causée par l'absorption du rayonnement solaire s'écrit

$$
\Delta T_{\mathrm{E}}=\frac{\alpha_{\mathrm{s}} \varphi_{\mathrm{s}}}{h} .
$$

Le coefficient d'absorption courte longueur d'onde $\alpha_{s}$ du revêtement de la paroi et du capteur a été déterminé par une expérience annexe.

On a trouvé : $\alpha_{s}=0.61$.

Avec cette valeur on en déduit le flux solaire reçu : $\varphi_{\mathrm{s}}=204 \mathrm{~W} / \mathrm{m}^{2}$. Une mesure directe de $\varphi_{\mathrm{s}}$ avec une cellule de rayonnement total nous a donné $\varphi_{\mathrm{s}}=20 \mathrm{S \textrm {W }} / \mathrm{m}^{2}$. L'accord est très satisfaisant.

\subsubsection{Essai d'interprétation des corrélations entre} $h_{\mathrm{c}}$ et $h_{0 \mathrm{c}}$ - - On utilise un résultat classique établi par Leveque. Pour ce faire on considère une plaque plane (Fig. 15a) à la température $T_{\mathrm{p}}$ balayée par un écoulement d'air turbulent à la température $T_{0}$. En $x \geqslant x_{0}$ on suppose que la température de la plaque passe de $T_{\mathrm{p}}$ à $T_{\mathrm{p}}+\delta T_{\mathrm{p}}$

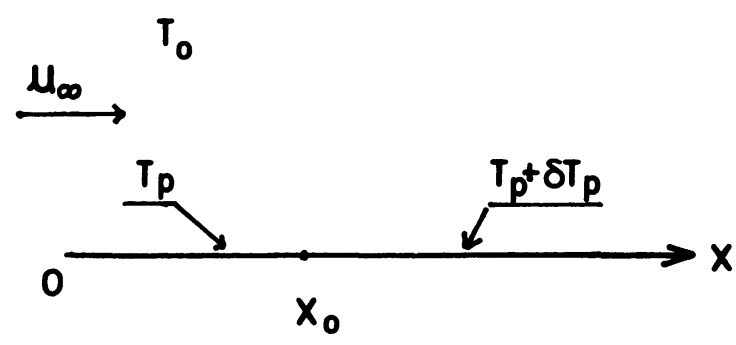

Fig. 15a. - Plaque plane à la température $T_{\mathrm{p}}$ constante entre 0 et $x_{0}$ avec perturbation de température à partir de $x=x_{0}$.

[Flat plate at constant temperature $T_{\mathrm{p}}$ between $x=0$ and $x=x_{0}$, with disturbance temperature after $x=x_{0}$.]

Ce problème est la superposition de deux problèmes (à cause de la linéarité des équations de transfert par convection) [3].

1. Le premier problème (Fig. 15b) correspond à la plaque plane à température constante balayée par de l'air à température $T_{0}$. On désignera par $h_{\mathrm{c}}$ le coefficient de convection, lié à cette couche limite thermique $\mathrm{CL}_{1}$, à l'abscisse $x$. Il est donné par le Nusselt en $x$ (l'écoulement turbulent débutant en $x=0$, formule de Colburn).

$$
N u_{x}=\frac{h_{\mathrm{c}} x}{\lambda}=0,029 P_{r}^{1 / 3} R e_{x}^{0,8}
$$

$P_{r}=v / a$ est le nombre de Prandtl de l'air ( $v$ et $a$ sont respectivement la viscosité cinématique et la diffusivité thermique de l'air). 


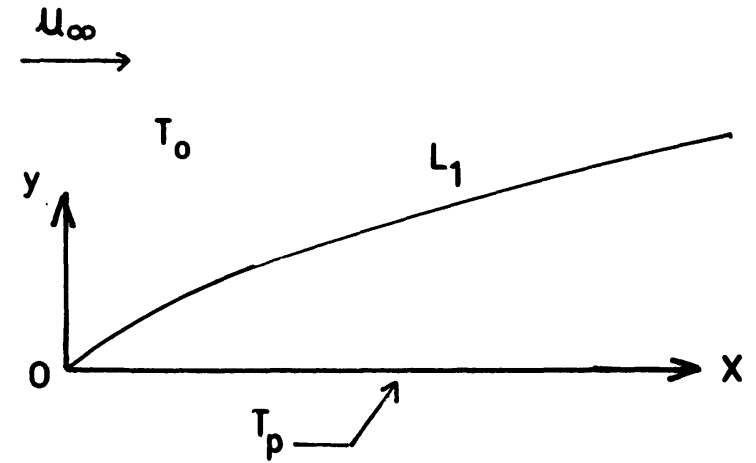

Fig. 15b. - Plaque plane à température constante $T_{\mathrm{p}}$.

[Flat plate at constant temperature $T_{\mathrm{p}}$.]

$R e_{x}=\frac{u_{\infty} x}{v}$ est le nombre de Reynolds de l'écoulement d'air en $x$.

2. Le second problème (Fig. 15c) a été étudié par Leveque [6], pour un écoulement laminaire. Si l'on désigne par $h_{0 c\left(x-x_{0}\right)}$ le coefficient local de convection lié à la couche limite thermique créée par cette perturbation thermique $\delta T_{\mathrm{p}}$ débutant en $x_{0}$, on a :

$$
h_{0 \mathrm{c}\left(x-x_{0}\right)}=\frac{\lambda}{0,893}\left(\frac{\gamma}{9 a\left(x-x_{0}\right)}\right)^{1 / 3}
$$

$\lambda$ étant la conductivité thermique de l'air, et $\gamma$ le gradient de vitesse au voisinage de la paroi, $\gamma=\partial u / \partial y$ qui est supposé constant.

Etant donné la faible dimension des capteurs, il nous semble justifié de penser que la couche limite thermique $\mathrm{CL}_{2}$ liée à la perturbation introduite par le capteur non équilibré est située entièrement dans la sous-couche visqueuse de l'écoulement $\left({ }^{1}\right)$.

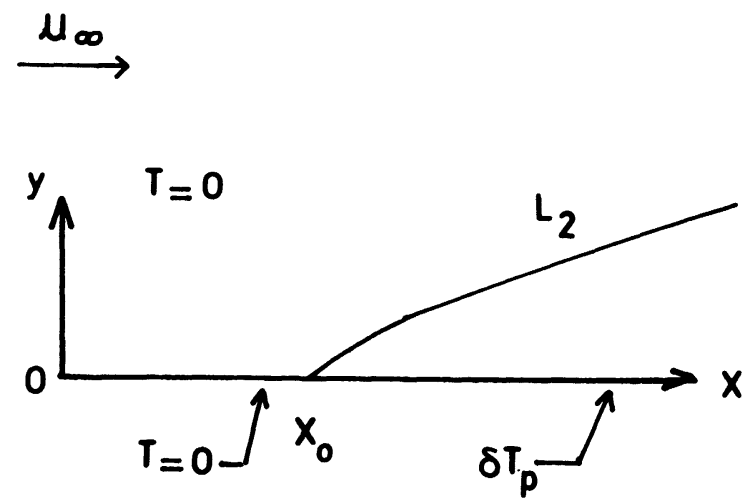

Fig. $15 c$. - Plaque plane à température $T=0$ entre 0 et $x_{0}$, à la température $T+\delta T_{\mathrm{p}}$ à partir de $x=x_{0}$.

[Flat plate at temperature $T=0$ between $x=0$ and $x=x_{0}$, at temperature $T+\delta T_{\mathrm{p}}$ after $x=x_{0}$.]

( $\left.{ }^{1}\right)$ Le rapport $r / x_{0}$ vaut 0,02 pour le capteur $A$ et 0,014 pour le capteur $B$; ainsi la contrainte pariétale varie très peu sur le capteur.
La formule établie par Leveque nous paraît utilisable pour déterminer le coefficient local de convection lié à cette perturbation.

La contrainte tangentielle due au frottement de l'air sur la plaque s'écrit :

$$
\tau_{\mathrm{p}}=\mu \frac{\partial u}{\partial y}=\mu \gamma \quad(\mu \text { viscosité dynamique de l'air })
$$

soit avec la relation (27)

$$
\tau_{\mathrm{p}}=\left(\frac{0,893}{\lambda}\right)^{3} 9 a\left(x-x_{0}\right) h_{\mathrm{oc}\left(x-x_{0}\right)}^{3}
$$

le coefficient de frottement en régime turbulent $C_{\mathrm{f}}$ est (formule de Blasius)

$$
C_{\mathrm{f}}=\frac{2 \tau_{\mathrm{p}}}{\rho u_{\infty}^{2}}=\frac{0,0592}{R_{\mathrm{ex}}^{0,2}} \quad(\rho \text { masse volumique de l'air }) \text {. }
$$

Des relations (28) et (29) on déduit le Nusselt lié à la couche limite $\mathrm{CL}_{2}$

$$
\begin{aligned}
N u_{0\left(x-x_{0}\right)}=\frac{h_{\mathrm{oc}\left(x-x_{0}\right)}\left(x-x_{0}\right)}{\lambda} & = \\
& =0,166 P_{\mathrm{r}}^{1 / 3} \frac{R e_{\left(x-x_{0}\right)}^{2 / 3}}{R e_{x_{0}}^{0,066}}
\end{aligned}
$$

d'où

$$
\begin{aligned}
h_{\mathrm{oc}\left(x-x_{0}\right)}=0,166 \operatorname{Pr}^{1 / 3} \cdot \lambda_{.} v^{-0,6} & \times \\
& \times \frac{x_{0}^{-0,066}}{\left(x-x_{0}\right)^{1 / 3}} u_{\infty}^{0,6} .
\end{aligned}
$$

Par intégration sur l'intervalle $\left[x_{0}, x_{0}+2 r\right]$ ( $r$ rayon du capteur), on en déduit le coefficient local moyen $h_{\mathrm{oc}}$ lié à la perturbation $\delta T_{\mathrm{p}}$.

Il est de la forme

$$
h_{\mathrm{oc}}=F(a, \lambda, v) \cdot G\left(x_{0}, 2 r\right) u_{\infty}^{0,6} .
$$

En éliminant la vitesse entre les relations (32 et 26), on en déduit les relations entre les coefficients de convection $h_{\mathrm{c}}$ lié à la couche limite $\mathrm{CL}_{1}$ et le coefficient $h_{0 \mathrm{c}}$ lié à la couche limite $\mathrm{CL}_{2}$ créé par la perturbation $\delta T_{\mathrm{p}}$

$$
h_{\mathrm{c}}=F_{1}(a, \lambda, v) G_{1}\left(x_{0}, 2 r\right) h_{0 \mathrm{c}}^{1,333}
$$

On retrouve une loi puissance analogue à la loi expérimentale, l'exposant étant cependant légèrement plus faible (1,33 au lieu de 1,36 pour le capteur $A$ et 1,42 pour le capteur $B$. Ce résultat nous semble très satisfaisant, compte tenu de la perturbation créée par la couche d'isolant $\delta r$, et dont il n'est pas tenu compte ici. Notons de plus que cette isolation est plus large pour le capteur B que pour A. 
7. Conclusion. - Nous avons voulu dans ce travail, exposer le principe de capteurs pariétaux permettant de caractériser globalement du point de vue énergétique les transferts superficiels entre une paroi avec revêtement hydrofuge et son environnement microclimatique en régime variable. De cette étude il ressort que ces transferts peuvent être caractérisés par la température de paroi $T_{\mathrm{p}}$ et par deux couples de données soit $h$ et $T_{\mathrm{E}}$, soit $h_{0}^{\prime}$ et $T_{\mathrm{c} 0}^{\prime}$.

$\mathrm{La}$ mesure directe de $h$ et $T_{\mathrm{E}}$ s'avère délicate car elle nécessite un capteur avec une régularisation très fine. Par contre la mesure de $h_{0}^{\prime}$ et $T_{\mathrm{c} 0}^{\prime}$ peut se faire avec un capteur présentant un simple dispositif de chauffage programmable : $h_{0}^{\prime}$ est obtenu par la loi de refroidissement d'un capteur soumis à une impulsion de flux, de durée très inférieure à la constante de temps du capteur, $T_{\mathrm{c} 0}^{\prime}$ est obtenue par l'enregistrement de la température d'un capteur dit inerte, c'est-à-dire sans dispositif de chauffage.

Le coefficient $h_{0}^{\prime}$ est plus grand que le coefficient $h$ : cette différence de valeur entre $h_{0}^{\prime}$ et $h$ provient des valeurs différentes des coefficients de convection pour la couche limite liée à la paroi $\left(h_{\mathrm{c}}\right.$ et $\left.h_{\mathrm{v}}\right)$ et pour la couche limite liée au capteur créant une perturbation thermique $\left(h_{0 \mathrm{c}}\right.$ et $\left.h_{0 \mathrm{v}}\right)$. Des considérations sur l'écoulement dynamique permettent de confirmer de manière très satisfaisante la corrélation expérimentale entre $h_{\mathrm{c}}$ et $h_{0 \mathrm{c}}$.

L'étude expérimentale montre que la constante de temps de ces capteurs, dans le domaine de vitesses étudiées, est de l'ordre de la minute, en fonction du coefficient de transfert $h$. Les capteurs seront donc capables de suivre avec un retard négligeable les fluctuations microclimatiques de durée supérieure ou égale à $4 \mathrm{~min}$, ce qui est satisfaisant; en effet les fluctuations de plus courte durée ne sont que très faiblement énergétiques et ne peuvent influencer de manière significative les transferts thermiques. Si on voulait les détecter, il faudrait utiliser des capteurs de plus petits diamètres.

\section{Bibliographie}

[1] BrunjaIl, C., Capteurs à impulsion pour la caractérisation des transferts thermiques entre une paroi et son environnement microclimatique. Société Française des Thermiciens, journées d'études, 10 mars 1981.

[2] JАKоB, Heat transfer (John Wiley and Sons, New York) 1959, vol. 1, p. 588.

[3] Fourcher, B., Transmission d'un flux thermique sinusoïdal à la frontière de séparation d'un solide et d'un fluide en écoulement permanent. Thèse Doctorat 3e cycle. Université de Nantes, 1974.

[4] Brunjail, C., Bardon, J. P., Capteurs pariétaux pour la caractérisation en régime variable des transferts thermiques paroi-environnement microclimatique. Rapport dans le cadre du plan construction (Cde no 78048), septembre 1979.

[5] Calvet, P., Liousse, F., Mesures locales de températures, pressions et vitesses au moyen de capteurs thermorésistants chauffés par impulsion. Revue Générale de Thermique no 114 (juin 1971).

[6] Knudsen, J. G., Katz, D. L., Fluid Dynamics and Heat Transfer (McGraw-Hill, New York) 1958, p. 363.

[7] Carslaw, H. S. and Jaeger, J. C., Conduction of Heat in Solids, $2 \mathrm{e}$ éd. (Oxford University Press, London) 1959. 